Montoya Grisales, N. E. y González Palacio, E. V. (septiembre-diciembre, 2019). Competencias TIC en docentes de nivel técnico y tecnológico. Un estudio de caso en un centro de formación del SENA. Revista Virtual Universidad Católica del Norte, (58), 74-95. doi: https://doi.org/10.35575/rvucn.n58a3

\title{
Competencias TIC en docentes de nivel técnico y tecnológico. Un estudio de caso en un centro de formación del SENA
}

ICT competences in teachers of technical and technological level. A case study at a SENA training center

\author{
Noelva Eliana Montoya Grisales \\ Magister en Ciencias de la Educación \\ Centro de Diseño y Manufactura del Cuero, Servicio \\ Nacional de Aprendizaje (SENA) \\ Colombia \\ elimontoya3@hotmail.com \\ Orcid: https://orcid.org/0000-0003-3267-6999
}

\author{
Enoc Valentín González Palacio \\ Doctor en Educación \\ Universidad de San Buenaventura \\ Colombia \\ enoc.gonzalez@usbmed.edu.co \\ Orcid: https://orcid.org/0000-0001-7708-7886
}

Recibido: 21 de febrero de 2019

Evaluado: 23 de mayo de 2019

Aprobado: 19 de junio de 2019

Tipo de artículo: Investigación científica y tecnológica.

\section{Resumen}

Las competencias TIC en la educación superior implican, para el docente, hacer uso de ellas desde su carácter instrumental; pero, también, implican comprender su función comunicativa y relacional. Estos asuntos fueron abordados desde una investigación mixta, en una muestra probabilística e intencionada de docentes de un centro de formación del SENA. Los resultados indican correlaciones positivas, entre moderadas y muy altas ( $r s: 0,56-0,09 ; p<0,01)$ en las competencias evaluadas, lo que permitió establecer que las competencias TIC no operan por separado y se constituyen en elementos complementarios entre sí; los datos cualitativos apoyan esta relación, y dejan entre leer que la capacitación por parte de las instituciones es fundamental en el proceso de adquisición y afianzamiento de estas competencias. Entre las principales conclusiones del estudio se destacan: las TIC son de gran ayuda en el proceso de enseñanza-aprendizaje, ya sea desde el componente comunicativo, como herramienta en el abordaje de contenidos, o como un medio didáctico; el proceso de adquisición y actualización de las competencias TIC, como elemento de la formación permanente y desarrollo profesional, son tanto responsabilidad de los docentes como de las instituciones, en la medida que el mejoramiento de la educación es un asunto de corresponsabilidad.

Palabras clave: Competencias del docente, Educación superior, Instructor, TIC. 


\section{Abstract}

ICT competences in higher education imply, for the teacher, to make use of them from their instrumental character; but, it also implies understanding their communicative and relational function. These issues were addressed from a mixed investigation, in a probabilistic and intentional sample of teachers from a SENA training center. The results indicate positive correlations, between moderate and very high ( $r s: 0.56-0.09, p<0.01$ ) in the evaluated competences, which allowed to establish that ICT competences do not operate separately and constitute complementary elements each; the qualitative data support this relationship, and allow us to understand that the training by the institutions is fundamental in the process of acquisition and consolidation of these competences. Among the main conclusions of the study are: ICT are of great help in the teaching-learning process, either from the communicative component, as well as a tool in the approach to contents and as a didactic medium; the process of acquiring and updating ICT competencies, as an element of ongoing training and professional development, are the responsibility of both teachers and institutions, in the sense that the improvement of education is a matter of co-responsibility.

Keywords: Teacher competences, Higher education, Instructor, ICT.

\section{| Introducción}

Un mundo cada vez más exigente, competitivo y ávido de información, ha hecho que el desarrollo de diferentes habilidades y destrezas sea una premisa del profesional actual; esta lógica hace parte también de la realidad docente en la educación superior. Para el caso de las tecnologías de la información y la comunicación -TIC-, existen una serie de habilidades y destrezas que son premisas internacionales, con respecto a lo que debe saber un docente; por ejemplo, la Organización de las Naciones Unidas para la Educación, la Ciencia y la Cultura -UNESCO- (2016), propone una serie de posibilidades frente a estas competencias, en sus “Estándares TIC desde la dimensión pedagógica", además de instancias administrativas, políticas y conceptuales.

Por su parte, el Ministerio de Educación Nacional de Colombia -MEN- (2009), estableció una serie de competencias genéricas y trasversales en educación superior, teniendo en cuenta los diferentes campos disciplinares; esto con el fin de tener una propuesta que no solo estuviera a la par de las exigencias globales, sino también como una misiva para el contexto local; se identificaron, entonces, los siguientes grupos de competencias genéricas para la educación superior: comunicación en lengua materna y otra lengua internacional, pensamiento matemático, ciudadanía, y ciencia, tecnología y manejo de la información.

Aparecen asociadas a la ciencia y a la investigación, las competencias en tecnología y comunicación, que las convierte no solo en una premisa relevante para el perfil docente, sino que las hace una competencia fundamental en los procesos de enseñanza-aprendizaje que están a su cargo; además de relacionarse con otros desempeños de los docentes de la educación superior, como la investigación, lo que de una u otra forma tendrá un efecto positivo y significativo en el sistema educativo, como se expresa en lo siguiente: 
El desarrollo de buenas prácticas pedagógicas apoyadas en las TIC constituye uno de los propósitos educativos de calidad propuestos por el Estado Colombiano y de cualquier sistema educativo que se encuentra permeado por los avances tecnológicos y el desarrollo propio de una sociedad globalizada. (Gamboa Suárez, Hernández Suárez y Prada Núñez, 2018, p. 259).

Igualmente, desde el punto de vista conceptual existen una serie de aportes de diferentes autores, frente a las TIC; de acuerdo con Sobrado, Ceinos y Fernández (2010), estas se definen como todas aquéllas herramientas que facilitan el desarrollo de tareas relacionadas con la adquisición, producción, almacenamiento, análisis y presentación de la información y de la comunicación, a través de diferentes lenguajes (p. 169); esta definición denota dos particularidades de las TIC; por un lado, su carácter instrumental como herramienta y, por el otro, su función de transmitir información y relacionarse con otros aspectos muy relevantes en la educación.

De esta manera, las TIC tienen un papel fundamental en la facilitación de la actualización y la comunicación (Hurtado Pérez y Vélez Holguín, 2010, p. 81); además, se reafirma el concepto de que las TIC son un conjunto de herramientas y recursos tecnológicos, físicos o digitales, que permiten a los sujetos tener acceso a la información, y comunicarse con otros; en Colombia, diferentes fuentes documentales sugieren como los principales recursos TIC, disponibles o usados por los docentes, los siguientes: procesadores de texto, hipertextos, editores de imágenes, de páginas Web, presentaciones multimedia, aplicaciones interactivas para el aprendizaje, chats, blogs, simuladores, correo electrónico, visitas, charlas, foros o conferencias virtuales (Mesa Agudelo, 2012; Pérez Cervantes y Saker, 2013). Al igual que para muchos docentes, para el caso de los instructores del SENA estas herramientas son una manera eficaz de impactar positivamente los procesos de enseñanza-aprendizaje.

Las TIC no son solo actuales, son, además, una necesidad en una organización de calidad, pues en los contextos educativos modernos son un requerimiento de los procesos formativos y una responsabilidad de los docentes que los atienden; de esta forma las TIC "son sinónimo de modernización, calidad, productividad, mejores servicios y apoyo a los procesos educativos" (Mesa Jiménez, 2012, p. 71).

Las TIC son un gran desafío en el campo educativo, pues los contenidos, metodologías y escenarios propiciados requieren de actualización constante, dado que los avances de los diferentes campos disciplinares y los procesos de enseñanza y aprendizaje, en los que se ven inmersos los actores del proceso educativo, son muy dinámicos y ágiles, lo que precisa estar al tanto de este dinamismo, "los desafíos educativos actuales son distintos a los del pasado, y tanto el papel como el lugar de la educación se han modificado" (Tedesco, 2011, p. 32); de esta manera, las competencias docentes, con respecto a las TIC, no son una opción, son un requerimiento del sistema educativo, o como dicen Gamboa Suárez et al. (2018):

La influencia significativa de las TIC en el aula ha traído como consecuencia la resignificación de la práctica pedagógica del docente y la necesidad de fortalecer sus competencias en relación con las habilidades tecnológicas para el desarrollo de buenas prácticas en su campo profesional. (p. 259). 
Pero la reflexión pedagógica frente al acto educativo y el uso de las TIC, no deben asumirse como una obligación contractual, deben entenderse como una tarea propia del rol del educador; el docente no puede estar a espalda de los cambios históricos, en las maneras de atender los procesos formativos, "históricamente las tecnologías de la información y la comunicación han venido y vienen acompañadas de un conjunto de cambios que obligan de una manera $u$ otra a revisar el hacer educativo" (Havery López, 2014, p. 12).

Las TIC como medio o herramienta, y su uso por parte de los docentes en interacción con los estudiantes, determinan otras dinámicas en los ambientes de aprendizaje, cosa que no escapa a la educación superior, pues las universidades están obligadas, dentro de sus procesos de acreditación, a tener y hacer uso de las TIC en sus diferentes misiones sustantivas, tal como el encuentro y prácticas pedagógicas, la reflexión del currículo, las didácticas activas, los métodos de enseñanza y la organización institucional (Vera Noriega, Torres Moran y Martínez García, 2014, p. 145); también, debe destacarse que una de las principales ventajas que tiene el uso de las TIC es que genera "nuevas posibilidades formativas al romper las barreras del tiempo y el espacio" (Hernández Ramos y Torrijos Fincias, 2019, p. 140).

Queda claro, entonces, que la educación superior está influenciada por las TIC, y es por esto que las instituciones y sus actores deben poseer las competencias requeridas para acceder a la información, almacenarla, procesarla y analizarla, y de esta manera generar conocimiento y transmitirlo a partir de las propias TIC; manifiestan Hernández y Torrijos (2019) que las metodologías de los docentes universitarios deben modificarse y actualizarse, haciendo uso de las TIC, pero con la principal intención de convertir al estudiante en el centro del aprendizaje (p. 141).

De esta manera, las TIC son una competencia que, desde el ámbito institucional y administrativo, tanto internacional como nacional, asi como desde lo conceptual y lo procedimental, deben poseer los docentes; pero ¿qué conocimiento tienen las instituciones educativas acerca de este aspecto?

El Centro del Diseño y Manufactura del Cuero, del Servicio Nacional de Aprendizaje (SENA), aborda este interrogante, con el fin de tener claro cómo los instructores se apropian de este tipo de competencias desde su componente instrumental, como herramienta didáctica en el proceso de enseñanza-aprendizaje, o como posibilidad de construcción de conocimiento científico en los procesos investigativos impulsados desde dicha institución.

Así las cosa, se pretendió dar a conocer el perfil de competencias TIC de los docentes, además de establecer cómo éstas son un recurso útil e indispensable en el proceso de enseñanza-aprendizaje, pero también que su potenciamiento está vinculado con los procesos de investigación y producción de conocimiento científico impulsados por la institución.

De esta manera, este proyecto dio respuesta al siguiente interrogante: ¿cuáles son las competencias que, en términos TIC, son usadas y requeridas por los instructores del Centro de Diseño y Manufactura del Cuero (CDMC) del SENA? 


\section{Método}

Esta investigación se enmarcó tanto por el método cuantitativo como cualitativo, por tanto, se estableció como un estudio multimétodo (Polit y Hungler, 2005), específicamente un diseño integrado por componentes, es decir, que los dos métodos usados se complementan.

Desde el punto de vista cuantitativo, el estudio fue no experimental descriptivo (Polit \& Hungler, 2005), pues pretendió identificar y describir las percepciones que, sobre competencias en términos de TIC, poseen los instructores del Centro del Diseño y Manufactura del Cuero del SENA, acudiendo a sus usos y conocimientos, como también a sus apreciaciones sobre las TIC en su función docente.

Frente a lo cualitativo, esta investigación complementó los resultados estadísticos desde una mirada interpretativa frente al uso de las TIC en los procesos de enseñanza aprendizaje de los instructores, por lo que se apoya en la investigación hermenéutica dialéctica, la cual es propuesta por Martínez (2010) para la comprensión de los fenómenos humanos.

En lo referido a la población objeto, los sujetos de indagación fueron los instructores del CDMC, que en su totalidad (N) corresponden a 105 sujetos; la muestra fue probabilística aleatoria simple (Grisales, 2001). Los parámetros para la selección de la muestra se presentan en la tabla 1, y las ecuaciones 1 y 2.

\section{Tabla 1.}

\section{Parámetros del muestreo}

\begin{tabular}{lc}
\hline Parámetro & Valor \\
\hline Confiabilidad (Z) & $95 \%(1,96)$ \\
Error & $5 \%$ \\
P & $50 \%$ \\
Q & $50 \%$ \\
Población (n) & 105 \\
\hline
\end{tabular}

Nota: elaboración propia. 


$$
\mathrm{n}=\frac{z_{\propto / 2}^{2} * \mathrm{pq}}{e^{2}+\frac{z_{\alpha / 2}^{2} * \mathrm{pq}}{N}}
$$

Ecuación 1.

\section{Fórmula para el cálculo de la muestra|}

El cálculo de la muestra arrojó un dato de 83 instructores (n), dado que es un valor superior al $10 \%$ de la población (N), este cálculo se ajusta (ñ) y se obtiene un valor de 46 sujetos; al finalizar el trabajo de campo se logró encuestar a 47 instructores, los cuales se escogieron al azar, utilizando una tabla de números aleatorios.

$$
\tilde{\mathrm{n}}=\frac{n}{1+\frac{n}{N}}
$$

\section{Ecuación 2.}

\section{Formula de ajuste de la muestra}

Para la indagación de naturaleza cualitativa se utilizó una muestra intencionada por conveniencia, teniendo en cuenta a docentes que fueran de áreas transversales y otros de áreas específicas, y que desempeñaran su labor tanto en el centro de formación (sede principal) como en sedes alternas; se entrevistaron a 4 docentes con estas características.

Para el proceso de recolección de la información, se atendieron las disposiciones bioéticas y la firma del consentimiento informado, por parte de los docentes indagados, las cuales son establecidas por la Resolución 8430 de 1993, del Ministerio de Salud Nacional.

Los instrumentos utilizados fueron: una encuesta con preguntas del perfil social y académico; el Cuestionario de perfil de competencias TIC, del docente del siglo XXI en educación superior (González et al., 2017) que consta de 40 ítems y que presenta una fiabilidad de 0,97 (Alfa de Cronbach); además, las entrevistas a profundidad.

En el procesamiento y análisis de los datos se tuvo en cuenta lo siguiente: los datos cuantitativos fueron digitados en una base de datos en Excel-2016, y posteriormente se importaron desde el programa SPSS v24 para el análisis estadístico; para el análisis cualitativo se utilizó la codificación axial, propuesta por Strauss y Corbin (2002), teniendo como ejes temáticos de las categorías (inductivas), las cuatro competencias en TIC, propuestas por González et al. (2017), a saber: reconocimiento de las TIC y sus elementos básicos, implementación de las TIC en el proceso educativo, optimización de la práctica educativa en términos de construcción de conocimiento, y conocimiento y usos de los recursos y espacios tecnológicos de la institución. Para la presentación de los diferentes testimonios, en cada una de las categorías, se identificó el número de la entrevista o documento primario (Ent-4), seguido del número del párrafo que fue citado (-14), y que fue generado por el programa Atlas-ti v7, en el cual se analizaron los datos cualitativos. 


\section{| Resultados}

Este estudio se realizó con 47 instructores del CDMC del SENA, cuyas características son las siguientes (ver tabla 2): un 57,4\% de ellos eran hombres; con respecto a la edad, la mediana fue de 38 años ( $c v=0,19$ ); en cuanto al nivel de formación, la mayoría de los instructores tienen especialización (40,4\%), seguido de pregrado (29,8\%), el nivel de maestría cuenta con un 17,0\% y los demás con formación tecnológica (12,8\%). Por otro lado, el mayor porcentaje, en tipo de vinculación, corresponde a prestación de servicios (76,6\%); frente al tiempo total laborado en el SENA, la mediana fue de 6,5 años (cv=0,78), y tiempo laborado en el CDMC, la mediana fue de 4,1 años $(c v=0,99)$.

Tabla 2

Frecuencias y estadísticos descriptivos de las variables del perfil social

\begin{tabular}{llll}
\hline Variable & Valores & N & $\%$ \\
\hline \multirow{2}{*}{ Género } & Femenino & 20 & 42,6 \\
& Masculino & 27 & 57,4 \\
& Tecnológica & 6 & 12,8 \\
Nivel de estudio & Pregrado & 14 & 29,8 \\
& Especialización & 19 & 40,4 \\
& Maestría & 8 & 17,0 \\
Tipo de vinculación laboral & Término indefinido & 2 & 4,3 \\
& Prestación de servicios & 36 & 76,6 \\
& Provisional & 9 & 19,1 \\
Edad (años) & Mediana -Coeficiente de variación (cv) \\
Tiempo laborado-SENA & $38-0,19$ & \\
(años) & $6,5-0,78$ & \\
Tiempo laborado - CDMC & & & \\
(años) & $4,1-0,99$ & \\
\hline
\end{tabular}

Nota: elaboración propia. $\mathrm{n}=47$.

Para la presentación de los resultados, se presenta la valoración cuantitativa de cada una de las dimensiones del perfil de las TIC de los instructores indagados, valoración que se hace a partir del cuestionario diseñado por González et al. (2017); igualmente, el análisis descriptivo se acompaña de los relatos cualitativos que ilustran las categorías (dimensiones) principales del estudio. 
En la primera dimensión: reconocimiento de las TIC y sus elementos básicos, la cual se refiere al dominio básico de algunas herramientas TIC, su utilización en el ambiente de aprendizaje y necesidad de actualización (ver tabla 3), se puede destacar que los instructores del SENA presentan la mayor valoración en el ítem donde consideran necesario y oportuno que las instituciones de educación superior ofrezcan capacitación para la actualización permanente en TIC para los docentes (media $=4,894 ; \mathrm{dt}=, 3117$ ); seguido por el item que considera que en el mundo actual (sociedad del conocimiento) es importante que los docentes de educación superior utilicen herramientas tecnológicas (media=4,766; dt=,4761); y, en tercer lugar, opinan que los docentes deben demostrar, en su modo de actuación, capacidades y competencias en TIC,


mente, para los instructores es fundamental estar a la vanguardia, en cuanto al conocimiento en TIC, y es esencial que la institución a la cual están vinculados ofrezca capacitaciones frente al tema; no obstante, en cuanto a lo que respecta al uso de las bases de datos bibliográficas para rastrear información documental, se presenta como el ítem que más bajo promedio presentó ( media=3,447; $\mathrm{dt}=1,1384$ ).

En el proceso de indagación cualitativa, los testimonios de la muestra indagada confirma lo descrito cuantitativamente; la categoría reconocimiento de las TIC y sus elementos básicos se hace a partir de dos asuntos; por un lado, reconocer que la formación del docente debe ser una primicia institucional, y es un espacio que es reclamado por ellos como elemento fundamental de su formación permanente; además, dado que las TIC son un elemento en constante evolución, esto lo hace un componente indispensable en la actualización profesional, tal como lo precisan algunos docentes: "Sería muy interesante si al menos una o dos veces al año nos permitan espacios de capacitación para ver nuevas tecnologías, nuevas herramientas existentes" (Ent-4-14), "Como dije anteriormente las TIC son un campo muy amplio, entonces todos los días ellas se están renovando y en esa medida nosotros tenemos que estar capacitándonos" (Ent-3-19).

De otra parte, las TIC no son solo importantes en la capacitación docente, en la medida que éstas son un recurso fundamental en la cotidianidad de las relaciones pedagógicas, sino que, además, son relevantes en la formación de los estudiantes; así las cosas, las TIC son tenidas en cuenta como herramienta, medio, proceso y contenido.

(...) para mí la implementación y el uso de las herramientas TIC es muy importante para los procesos formativos, el uso de plataformas diferentes para realizar actividades con los aprendices, es decir, la formación de hoy no se puede limitar solo al tablero, papel o lápiz, sino tener diferentes mecanismos no solo para evaluar los aprendices, sino para enseñarles. (Ent-4-7).

Los anteriores resultados ya han sido reportados por otros estudios; tal es el caso de Valdés Cuervo, Angulo Armeta, Urías Martínez, García López y Mortis Lozoya (2011), quienes encontraron que "existen altas necesidades de capacitación de manera global en el uso de las TIC" (p. 219), y que esa formación debería estar enfocada en aspectos pedagógicos, lo cual llevaría a una mejor práctica docente. Se hace fundamental, entonces, que exista una capacitación, pero también es claro que esta no puede estar basada meramente en lo instrumental, es decir, solo en el manejo de programas, sino que "es necesario que los docentes sean capacitados en cuestiones que tienen que ver con la aplicación de TIC en el aula, y sobre todo ésta debe centrarse en cómo emplearlas de manera didáctica" (Almirón y Porro, 2014, p. 22). 
Algo que se destaca y se infiere de los resultados anteriores, es cómo el proceso de actualización no es solo una responsabilidad del docente, sino que el centro formativo debe ser coparticipe en este proceso, pues cada institución posee unas características, necesidades y sujetos particulares; en esta medida la actualización de la misma, de sus docentes y estudiantes, debe estar anclada a las revisiones periódicas de los planes de estudio y el currículo en general, lo cual no solo se debe hacer por una premisa reglamentaria, sino también un deber social y educativo para con el contexto y los sujetos que lo integran.

Tabla 3

Estadisticos descriptivos sobre el reconocimiento de las TIC y sus elementos básicos

\begin{tabular}{|c|c|c|}
\hline Ítems & Media & Desv. típ. \\
\hline $\begin{array}{l}\text { Hago uso de las bases de datos bibliográficas para rastrear } \\
\text { información documental }\end{array}$ & 3,447 & 1,1384 \\
\hline $\begin{array}{l}\text { Tengo un manejo adecuado de herramientas de office (Word, } \\
\text { Excel, power point, entre otros) }\end{array}$ & 4,340 &, 7306 \\
\hline $\begin{array}{l}\text { Hago uso de las TIC para evaluar el desarrollo de aprendizaje } \\
\text { de estudiantes y agilizar los procesos de calificación y entrega } \\
\text { de notas }\end{array}$ & 4,468 &, 5044 \\
\hline $\begin{array}{l}\text { Utilizo las TIC para obtener información sobre temáticas } \\
\text { relacionadas con mi desempeño docente y las tendencias } \\
\text { educativas }\end{array}$ & 4,234 &, 8899 \\
\hline $\begin{array}{l}\text { Las TIC me facilitan la comunicación con el estudiante y para } \\
\text { ello hago uso del WhatsApp, correo electrónico, Facebook, } \\
\text { entre otros }\end{array}$ & 4,426 &, 9265 \\
\hline $\begin{array}{l}\text { Hago uso de una página web, blog u otra herramienta donde } \\
\text { comparto información de la asignatura y el trabajo con los } \\
\text { estudiantes }\end{array}$ & 3,702 & 1,1594 \\
\hline $\begin{array}{l}\text { Considero que el uso de herramientas tecnológicas en el } \\
\text { proceso de enseñanza potencia el aprendizaje de los estudiantes }\end{array}$ & 4,638 &, 5682 \\
\hline $\begin{array}{l}\text { Considero que en el mundo actual (Sociedad del conocimiento) } \\
\text { es importante que los docentes de educación superior utilicen } \\
\text { herramientas tecnológicas (TIC) }\end{array}$ & 4,766 &, 4761 \\
\hline $\begin{array}{l}\text { Considero necesario y oportuno que las instituciones de } \\
\text { educación superior ofrezcan capacitación para la actualización } \\
\text { permanente en TIC para los docentes }\end{array}$ & 4,894 &, 3117 \\
\hline $\begin{array}{l}\text { En mi opinión los docentes deben demostrar en su modo de } \\
\text { actuación capacidades y competencias en TIC, para potenciar } \\
\text { habilidades tecnológicas en los estudiantes }\end{array}$ & 4,702 &, 5483 \\
\hline $\begin{array}{l}\text { Soy un docente del siglo XXI y por lo tanto hago uso para el } \\
\text { proceso de enseñanza aprendizaje de: almacenamiento en la } \\
\text { "nube" (Google Drive, Dropbox, Skype etc.), Blogs, } \\
\text { plataformas virtuales, bases de datos, wikis, bibliotecas } \\
\text { virtuales, redes sociales, }\end{array}$ & 4,447 &, 6189 \\
\hline
\end{tabular}

Nota: elaboración propia. $\mathrm{n}=47$ 
En cuanto a la dimensión de implementación de las TIC en el proceso educativo, que se refiere al uso de las TIC para la planeación, ejecución y evaluación del proceso formativo (ver tabla 4), el aspecto que presentó mayor promedio, es en el que los instructores consideran que es importante utilizar las TIC para visualizar la estructura de los contenidos en las asignaturas que orientan (media=4,511; $d t=, 6875$ ), y que para el desarrollo de sus clases tienen en cuenta el uso de herramientas, tales como el video beam, televisor, computador, entre otras (media=4,511; $\mathrm{dt}=, 7185)$; para el caso particular del SENA, una de las herramientas más referenciadas es el uso de "la plataforma blackboard" (Ent-3-11). Cabe señalar que el ítem que presentó menor promedio es el de rastreo de bases de datos bibliográficas en el proceso de enseñanza aprendizaje, como una actividad permanente en las actividades académicas (media=3,660; $\mathrm{dt}=$ 1,0060).

Los docentes manifiestan en sus testimonios, que la implementación de las TIC en las aulas como herramienta, es de muy buena aceptación por parte de los estudiantes, pues ayuda al dinamismo y al orden de la clase; además, posibilita una forma de almacenar y comunicar información; por otra parte, los docentes, en sus maneras de referirse a las TIC, suelen asociarlas al uso de software, los más comunes son los programas ofimáticos o del paquete office; aunque dados los diferentes programas y procesos formativos que se ofrecen en el SENA, algunos docentes presentan dominios de software específicos, acordes a sus perfiles de formación e instrucción.

Pues, es que se vuelve una herramienta necesaria en este momento, porque todo el tiempo a los muchachos les llama mucho más la atención cuando se hacen presentaciones con imágenes, cuando son interactivas y no solo que uno se quede en un tablero o haciendo carteleras, entonces si es muy importante el manejo de las TIC, porque se pueden afianzar más los conocimientos y le ayuda a uno para tener un orden de toda su sesión y pues también los muchachos tienen la oportunidad de tener esas nuevas memorias para estudiar o para repasar lo visto en clase, entonces se vuelve más fácil toda esa parte de la comunicación. (Ent-1-20).

Manejo obviamente softwares específicos para el área de diseño como son Corel, ilustrator, Photoshop, rhinoceros, AutoCAD, entonces desde esa parte en las TIC está bien, y desde la parte de office, también generalmente hacemos muchas presentaciones. (Ent-1-8).

Se presenta otro elemento en la descripción de la implementación de las TIC en el proceso educativo, que funge más como un requerimiento que como un componente, y es el hecho de que, dada la dinámica formativa descentralizada de la institución, no siempre es posible garantizar el acceso y uso de herramientas TIC en los procesos formativos de los estudiantes, tal como se aprecia en el siguiente testimonio: "y lo otro es que no todos los grupos tienen el acceso a las herramientas mínimas como computador o internet, porque hay muchos grupos que están en sedes alternas y no se cuenta con las herramientas todo el tiempo" (Ent-4-10).

Lo mencionado anteriormente se relaciona con los resultados hallados por López, Correa y Rojas-Bahamón (2017), en cuanto a que, cuando los docentes describen el uso de las TIC, se refieren a la utilización de herramientas, como el computador, video proyector, televisor, paquete de Microsoft office, entre otros y "se resalta su implementación en el aula como potencial herramienta educativa de apoyo a la enseñanza; esto por cuanto mejora las condiciones de las prácticas en relación con la competencia del profesor"(p. 269). 
No obstante, en la misma investigación se reporta que para la población estudiada los entornos virtuales de aprendizaje no emergen con propiedad en el escenario educativo. Por el contrario, en el SENA, las TIC sí son un objeto muy relevante en el proceso formativo, dado que en esta institución se busca una interacción entre la formación presencia y virtual; frente a esto, aportan Fernández Cruz, Fernández Díaz y Rodríguez Mantilla (2018) que este tipo de herramientas de comunicación y trabajo cooperativo permiten obtener un contexto de infraestructuras imprescindibles para desarrollar habilidades y competencias digitales, tanto en los profesores como en los estudiantes.

De los resultados anteriores, se puede interpretar cómo las TIC fungen como medio en el proceso de enseñanza - aprendizaje; poseer un equipo o herramienta tecnológica puede llegar a marcar una buena relación o conexión con los estudiantes, quienes ven, con buena manera, que un docente prepare y oficie su clase haciendo uso de estas herramientas; en este orden de ideas, esto también se convierte en un reto institucional, en la medida que el centro formativo debe garantizar una serie de recursos, equipos y espacios que posibiliten esta acción.

Tabla 4

Estadísticos descriptivos sobre la implementación de las TIC en el proceso educativo

\begin{tabular}{lll}
\hline Items & Media & Desv. típ. \\
\hline $\begin{array}{l}\text { El rastreo de bases de datos bibliográficas en el proceso de } \\
\text { enseñanza aprendizaje es una actividad permanente dentro de las } \\
\text { actividades académicas que acompaño con los estudiantes. }\end{array}$ & 3,660 & 1,0060 \\
$\begin{array}{l}\text { Utilizo bases de datos, webs, plataformas y bibliotecas virtuales } \\
\text { para la búsqueda de información actualizada en cuanto a nuevas }\end{array}$ & 4,021 & 1,0106 \\
tendencias educativas mediadas por TIC. & \\
$\begin{array}{l}\text { Considero importante utilizar las TIC para visualizar la estructura } \\
\text { de los contenidos en las asignaturas que oriento. } \\
\text { Tengo en cuenta, para el desarrollo de mis clases, el empleo de }\end{array}$ & 4,511 &, 6875 \\
herramientas como: video beam, televisor, video cámaras, \\
grabadoras, computador, entre otras. \\
$\begin{array}{l}\text { Tengo en cuenta sugerencias dadas por grupos de apoyo, colegas y } \\
\text { estudiantes para implementar herramientas que faciliten el proceso } \\
\text { de enseñanza aprendizaje. }\end{array}$ & 4,489 &, 7185 \\
$\begin{array}{l}\text { Utilizo herramientas TIC como: office, plataformas virtuales, bases } \\
\text { de datos, bibliotecas virtuales, webs en la planeación de mis } \\
\text { actividades académicas. }\end{array}$ & 4,426 &, 6550 \\
$\begin{array}{l}\text { Empleo las TIC para proveer retroalimentación a los estudiantes a } \\
\text { partir de su proceso de evaluación. }\end{array}$ & 4,426 &, 6509 \\
$\begin{array}{l}\text { Planteo estrategias con el empleo de TIC para la presentación de } \\
\text { contenidos que consideren los ritmos y estilos de aprendizajes de } \\
\text { los estudiantes. }\end{array}$ & 4,255 &, 8462 \\
$\begin{array}{l}\text { Evalúo la efectividad de mis prácticas pedagógicas apoyadas en } \\
\text { TIC para la transmisión de información, contenidos y búsqueda de } \\
\text { información de calidad. }\end{array}$ & 4,128 &, 7107 \\
\hline Nota: elaboración propia. n=47 & & \\
\hline
\end{tabular}


Frente a la tercera dimensión, optimización de la práctica educativa en términos de construcción de conocimiento a partir de las TIC (ver tabla 5), en general todos los ítems presentan promedios por encima de 4,3, pero los que mayor valoración presentan son: las TIC como herramientas fundamentales en la producción de textos científicos y en la formación de nuevos investigadores (media=4,702; dt=,5071), y, en el mismo orden de ideas, el ítem de TIC como herramientas fundamentales para la generación de productos relacionados con la apropiación social del conocimiento (media=4,681; $\mathrm{dt}=, 5153$ ); esto establece las TIC como un punto de referencia, no solo como un criterio para la producción de nuevo conocimiento y la emergencia de nuevos investigadores, sino también como una herramienta útil para la generación de productos que se dan a conocer a la comunidad académica en general.

De acuerdo con los datos cualitativos, los docentes presentan una percepción muy positiva frente a la utilidad las TIC en el proceso de producción y dinámica investigativa; no obstante, reclaman de la institución la implementación de software, requeridos para procesos investigativos. "Pues definitivamente, yo pienso que algunos softwares en temas relacionados en investigación, ser un poquito más robusto en el tema de base de datos bibliográficos, que la verdad si estamos un poquito quedados" (Ent-2-20).

Se aprecia, entonces, que los docentes reclaman mucha más actualización en el tema de bases de datos bibliográficas, lo que contrasta con la poca valoración que hacen los docentes en el uso de las bases de datos bibliográficas para rastrear información documental (ver tabla 3); sin embargo, esto no concuerda con un estudio realizado en universidades del caribe colombiano (Said-Hung et al., 2015, p. 286), en donde se encontró que, en términos de uso, uno de los recursos tecnológicos con más predominio y arraigo son las "bases de datos especializadas", al igual que el correo electrónico, entre otros.

A pesar de las percepciones positivas frente a las TIC, como una posibilidad y una herramienta de gran valor para la producción de conocimiento científico, esta postura positiva no necesariamente contrastaría con un cúmulo de producciones científicas, sobre todo para el caso de productos de nuevo conocimiento. Los docentes pertenecientes al centro formativo indagado, dan privilegio a productos referidos a la apropiación social del conocimiento y la investigación formativa. 
Tabla 5

Estadisticos descriptivos Optimización de la práctica educativa en términos de construcción de conocimiento a partir de las TIC

$+$

\begin{tabular}{lcc}
\hline Ítems & Media & Desv. típ. \\
\hline $\begin{array}{l}\text { Utilizo las herramientas TIC para favorecer la construcción } \\
\text { de conocimiento de tipo activo, autónomo y colaborativo }\end{array}$ & 4,362 &, 6402 \\
$\begin{array}{l}\text { Consideró importante utilizar las TIC para optimizar la } \\
\text { práctica educativa en términos de la construcción de }\end{array}$ & 4,638 &, 5286 \\
$\begin{array}{l}\text { conocimiento } \\
\text { Las TIC son herramientas fundamentales en la producción }\end{array}$ & 4,702 &, 5071 \\
de textos (Artículos, libros) científicos & \\
$\begin{array}{l}\text { Las TIC son herramientas fundamentales para la generación } \\
\text { de productos relacionados con la apropiación social del }\end{array}$ & 4,681 &, 5153 \\
$\begin{array}{l}\text { conocimiento (Participación y formulación de eventos } \\
\text { académicos) }\end{array}$ & \\
$\begin{array}{l}\text { Las TIC son herramientas fundamentales en la formación } \\
\text { de nuevos investigadores }\end{array}$ & 4,702 &, 5071 \\
$\begin{array}{l}\text { Las TIC son herramientas fundamentales en el diseño de } \\
\text { producto referidos a nuevas tecnologías e invenciones }\end{array}$ & 4,660 &, 5224 \\
$\begin{array}{l}\text { Hago uso de las TIC para asesorias académicas } \\
\text { El divulgar y compartir mis conocimientos sobre la } \\
\text { implementación de las TIC a través de medios formales e } \\
\text { informales es relevante en mis cursos }\end{array}$ & 4,340 &, 7388 \\
$\begin{array}{l}\text { Las TIC permiten mayor flexibilidad de espacio, tiempo y } \\
\text { manejo de recursos. }\end{array}$ & 4,532 &, 6545 \\
\hline
\end{tabular}

Nota: elaboración propia. $\mathrm{n}=47$

En lo referente a la cuarta dimensión, conocimiento y uso de los recursos y espacios tecnológicos de la institución (ver tabla 6), la mejor valoración la tiene el ítem de utilización de las plataformas virtuales para la administración de los cursos asignados (media=4,511; dt=,7185), seguido por: considero imprescindible el uso de las TIC en mi labor docente (media=4,404; $\mathrm{dt}=$,7419). Esto se da claramente porque uno de los objetivos del SENA es que, durante el desarrollo curricular, los instructores se apropien de las herramientas que la institución posee, y que parte de la orientación de la formación se haga a través de ambientes virtuales de aprendizaje, en este caso específico la plataforma blackboard. Por otra parte, el ítem con menor

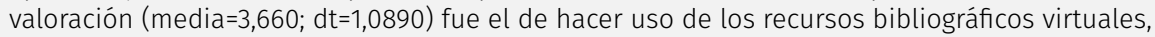
de los cuales dispone la institución para la labor docente; en otras palabras, los instructores conocen las herramientas, recursos y espacios tecnológicos de la institución, pero no necesariamente hace un uso de todos ellos.

Frente a lo anterior, los docentes del SENA manifiestan que utilizan los espacios y recursos TIC de la institución, incluso la mayoría de ellos manifiesta que tienen la disponibilidad para realizar cursos de actualización; no obstante, no todos los espacios en los cuales se imparte información, como, por ejemplo, en las sedes alternas, es posible tener disponibles todas las herramientas y recursos que se deberian. “(...) al menos en la sede principal tenemos una buena conexión a internet y computadores disponibles, creo que es un primer paso y en las sedes alternas procurar que se cuenten con las herramientas" (Ent-4-12). 
(...) entonces digamos que va a depender mucho del ambiente y a veces es muy difícil porque (...) en muchas de las actividades que uno tiene planificadas no se pueden hacer, porque solo hay una sala de sistemas y entonces es muy difícil acceder a ella y la prioridad es para otras competencias. (Ent-2-11)

Las anteriores dificultades, no solo se presentan en términos de espacio o de hardware, sino también en términos de las posibilidades de uso de software, tal como se aprecia en lo siguiente:

(...) eso ha cambiado mucho, ahorita dicen que se trabaja por red, entonces la red no está funcionando porque no están gestionando adecuadamente los programas y nos quedamos con muy poquitas licencias, entonces no todos los computadores tienen la licencia y ahí si es muy complicado. (Ent-1-13).

El uso apropiado de las TIC, como se ha podido apreciar en líneas anteriores, requiere de un docente que las reconozca, las use y se actualice en ellas; no obstante, también es indispensable que las instituciones educativas implementen y cuenten con los recursos y espacios necesarios para ello; además, deben incentivar su uso en el proceso docente, la extensión y la investigación.

Tabla 6

Estadísticos descriptivos sobre el conocimiento y usos de los recursos y espacios tecnológicos de la institución

\begin{tabular}{lcc}
\hline Ítems & Media & Desv. típ. \\
\hline $\begin{array}{l}\text { Conozco los espacios destinados para el uso de TIC en la práctica } \\
\text { docente de la institución universitaria donde laboro }\end{array}$ & 4,149 &, 8841 \\
$\begin{array}{l}\text { Conozco los diferentes recursos TIC de los cuales dispone la } \\
\text { institución universitaria para la práctica docente }\end{array}$ & 4,277 &, 7431 \\
$\begin{array}{l}\text { Utilizo los espacios destinados para el uso de TIC con los que } \\
\text { cuenta la universidad para las clases que tengo a cargo }\end{array}$ & 4,149 &, 9084 \\
$\begin{array}{l}\text { Hago uso de los diferentes recursos tecnológicos de la institución } \\
\text { para mis clases }\end{array}$ & 4,298 &, 8576 \\
$\begin{array}{l}\text { Utilizo plataformas virtuales para la administración de los } \\
\text { cursos que me son asignados }\end{array}$ & 4,511 &, 7185 \\
$\begin{array}{l}\text { Me capacito y actualizo sobre el uso de las TIC en la labor } \\
\text { docente }\end{array}$ & 4,174 &, 8247 \\
$\begin{array}{l}\text { Considero imprescindible el uso de las TIC en mi labor docente } \\
\begin{array}{l}\text { Considero que la universidad debe incluir en los procesos de } \\
\text { formación de sus estudiantes las TIC }\end{array}\end{array}$ & 4,404 &, 7419 \\
$\begin{array}{l}\text { Hago uso de los recursos bibliográficos virtuales de los cuales } \\
\text { dispone la universidad para mi labor docente }\end{array}$ & 3,660 & 1,0890 \\
$\begin{array}{l}\text { Utilizo el correo institucional para comunicarme con los } \\
\text { diferentes miembros de la comunidad académica }\end{array}$ & 4,298 &, 9536 \\
$\begin{array}{l}\text { Considero de gran importancia la participación en redes y } \\
\text { pertenezco a alguna de ellas }\end{array}$ & 4,106 & 1,0474 \\
\hline
\end{tabular}

Nota: elaboración propia. $n=47$ 
Un elemento que emerge de los datos cualitativos es que los docentes reconocen, por un lado, que efectivamente poseen conocimientos relativos al uso e implementación de las TIC en los procesos formativos que tienen a cargo; pero, también, manifiestan que algunos de ellos requieren procesos de actualización o de perfeccionamiento frente a las TIC, y no solo por ser un elemento de formación personal, sino que por la misma dinámica de las TIC, en términos de avances y evolución de la tecnología, esto se hace imprescindible; en pocas palabras, es una premisa de formación continua, es decir, es un reto del docente actual. "(...) a mí me hace falta, reconozco que me falta, yo puedo tener una base, pero es una base muy pequeñita, me parece vital ampliarlo" (Ent-4-17).

(...) una mayor capacitación en el área de informática, porque todos los días ella se está renovando, hay por ejemplo programas de mi área como por ejemplo nasus, TCO y muchas más, que en esas si me falta un poco más de formación. (Ent-2-9).

En términos generales, se encontró para este grupo de instructores, que en su mayoría presentan niveles superiores en las competencias TIC (ver tabla 7), destacándose la referida a la optimización de la práctica educativa en términos de construcción de conocimiento a partir de las TIC, la cual presentó como principal valoración el nivel superior en un 95,7\% de los casos; las otras dimensiones del perfil TIC, reconocimiento de las TIC y sus elementos básicos, implementación de las TIC en el proceso educativo, y conocimiento y usos de los recursos y espacios tecnológicos de la institución, presentaron también una mayor frecuencia en la valoración superior, con porcentajes por encima del $87 \%$. Lo anterior, muestra que los instructores tienen conocimiento, hacen uso y tienen prácticas efectivas tanto en relación con las TIC en el proceso de enseñanza aprendizaje, como en los asuntos investigativos, de producción de conocimiento y en la formación. Resultados muy similares al estudio presentado por Montoya Grisales, Mosquera Bermúdes, Pérez Martínez y Arroyave Giraldo (2018), en una universidad de la ciudad de Medellín, en donde todas las competencias TIC, también tuvieron calificaciones superiores, pero se destacaron las referidas al reconocimiento de las TIC y sus elementos básicos, y a la implementación de las TIC en el proceso educativo, con porcentajes de 91,5. De igual manera, Tello Díaz y Cascales Martínez (2015) encontraron que el "nivel de conocimientos que el profesorado dice tener respecto a contenidos y aplicaciones TIC es alto" (p. 386), utilizando herramientas de comunicación, diferentes metodologías y estrategias de las TIC. 


\section{Tabla 7}

Frecuencias de las calificaciones de las dimensiones y el perfil general de las competencias TIC en educación superior

\begin{tabular}{lccc}
\hline \multicolumn{1}{c}{ Dimensión } & Básico & Intermedio & Superior \\
\hline $\begin{array}{l}\text { Calificación Reconocimiento de } \\
\text { las TIC y sus elementos básicos }\end{array}$ & 0 & 6,4 & 93,6 \\
$\begin{array}{l}\text { Calificación Implementación de } \\
\text { las TIC en el proceso educativo }\end{array}$ & 0 & 12,8 & 87,2 \\
$\begin{array}{l}\text { Calificación Optimización de la } \\
\text { práctica educativa en términos de } \\
\text { construcción de conocimiento a } \\
\text { partir de las TIC }\end{array}$ & 0 & 4,3 & 95,7 \\
$\begin{array}{l}\text { Calificación Conocimiento y usos } \\
\text { de los recursos y espacios } \\
\text { tecnológicos de la institución }\end{array}$ & 2,1 & 6,4 & 91,5 \\
\begin{tabular}{l} 
Calificación TIC general \\
\hline
\end{tabular} & 0 & 4,3 & 95,7 \\
\hline
\end{tabular}

Nota: elaboración propia. $n=47$

Al correlacionar las diferentes dimensiones del perfil TIC (ver tabla 8), se encuentran relaciones positivas moderadas $(r h o=0,4-6)$ y altas $(r h o=0,6-0,8)$ entre ellas, lo que indica que, entre más alta sea una competencia en TIC las otras también lo serán; igualmente, se presentan asociaciones estadísticamente muy significativas entre las dimensiones valoradas $(p=0,000)$, lo que muestra además un alto grado de asociación entre este tipo de competencias; por ejemplo, tener un alta implementación de la TIC en el proceso educativo, puede significar optimizar la práctica educativa, en términos de construcción de conocimiento, y mejorar el uso y conocimientos de los recursos de la institución.

La dimensión TIC que presentó valores más altos de correlación con las otras dimensiones ( $r h o=0,630, r h o=0,713, r h o=0,796)$ y con el valor total del perfil TIC (rho=0,903) fue la dimensión de conocimiento y usos de los recursos y espacios tecnológicos de la institución; por tanto, entre mayor se presenta el conocimiento de los espacios y recursos TIC de la institución, mayor es el reconocimiento de las TIC, su implementación en las clases y su optimización en los procesos de investigación; además de presentarse un aumento en la valoración general de las competencias TIC, correlaciones que además presentaron asociaciones estadísticamente muy significativas $(p<0,000)$. 


\section{Tabla 8}

Correlaciones entre las dimensiones de las competencias TIC

†

\begin{tabular}{|c|c|c|c|c|c|c|}
\hline Dimensiones & & $\begin{array}{l}\text { Reconocimient } \\
\text { o de las TIC y } \\
\text { sus elementos } \\
\text { básicos }\end{array}$ & $\begin{array}{l}\text { Implementació } \\
\mathrm{n} \text { de las TIC en } \\
\text { el proceso } \\
\text { educativo }\end{array}$ & $\begin{array}{c}\text { Optimizació } \\
\text { n de la } \\
\text { práctica } \\
\text { educativa en } \\
\text { términos de } \\
\text { construcción } \\
\text { de } \\
\text { conocimient } \\
\text { o a partir de } \\
\text { las TIC }\end{array}$ & $\begin{array}{l}\text { Conocimient } \\
\text { o y usos de } \\
\text { los recursos } \\
\text { y espacios } \\
\text { tecnológicos } \\
\text { de la } \\
\text { institución }\end{array}$ & $\begin{array}{l}\text { Valor } \\
\text { TIC } \\
\text { total }\end{array}$ \\
\hline $\begin{array}{l}\text { Reconocimient } \\
\text { o de las TIC y }\end{array}$ & $\begin{array}{l}\mathrm{r} \\
\mathrm{s}\end{array}$ & 1,000 & $644^{* *}$ &, $560^{* *}$ &, $630^{* *}$ &, $789^{*}$ \\
\hline $\begin{array}{l}\text { sus elementos } \\
\text { básicos }\end{array}$ & $\mathrm{p}$ & &, 000 &, 000 &, 000 &, 000 \\
\hline $\begin{array}{l}\text { Implementació } \\
\mathrm{n} \text { de las TIC en }\end{array}$ & $\begin{array}{l}r \\
s\end{array}$ &, $644^{* *}$ & 1,000 &, $731^{* *}$ &, $713^{* *}$ & ${ }^{873^{*}}$ \\
\hline $\begin{array}{l}\text { el proceso } \\
\text { educativo }\end{array}$ & $\mathrm{p}$ &, 000 & & ,000 &, 000 & ,000 \\
\hline $\begin{array}{l}\text { Optimización } \\
\text { de la práctica } \\
\text { educativa en } \\
\text { términos de }\end{array}$ & $\begin{array}{l}r \\
s\end{array}$ &, $560^{* *}$ &, $731^{* *}$ & 1,000 &, $796^{* *}$ & $879^{*}$ \\
\hline $\begin{array}{c}\text { construcción de } \\
\text { conocimiento a } \\
\text { partir de las } \\
\text { TIC }\end{array}$ & $\mathrm{p}$ &, 000 &, 000 & &, 000 &, 000 \\
\hline $\begin{array}{l}\text { Conocimiento } \\
\text { y usos de los } \\
\text { recursos y }\end{array}$ & $\begin{array}{l}r \\
s\end{array}$ &, $630^{* *}$ &, $713^{* *}$ &, $796^{* *}$ & 1,000 &, $903^{\circ}$ \\
\hline $\begin{array}{c}\text { espacios } \\
\text { tecnológicos de } \\
\text { la institución }\end{array}$ & $\mathrm{p}$ & ,000 &, 000 &, 000 & &, 000 \\
\hline \multirow[t]{2}{*}{ Valor TIC total } & $\begin{array}{l}r \\
s\end{array}$ &, $789^{* *}$ & $873^{* *}$ &, $879^{* *}$ &, $903^{* *}$ & 1,000 \\
\hline & $\mathrm{p}$ &, 000 &, 000 &, 000 &, 000 & \\
\hline
\end{tabular}

Nota: elaboración propia. ${ }^{* *}$. La correlación es significativa al nivel 0,01 (bilateral). (rs) Rho de Spearman 
Al relacionar y asociar las diferentes dimensiones de las competencias TIC con las variables del perfil social y académico, no se presentaron correlaciones ( $r s$ ) ni asociaciones estadísticamente significativas ( $\mathrm{rs}, \mathrm{x} 2$ ) entre ellas, por lo cual el nivel de competencias TIC en los instructores del SENA no presente dependencia estadística, con variables como el género, la edad, el tipo de contratación, la experiencia profesional ni el nivel de estudio.

\section{| Discusión}

El reconociendo de las TIC y sus diferentes componentes básicos son de gran importancia para los docentes indagados, pues no solo identifican las diferentes herramientas tecnológicas, sino que también manifiestan hacer uso de ellas de forma apropiada; al igual que el estudio realizado por Gamboa Suárez et al. (2018) en donde los docentes “afirman poseer una buena capacidad en el uso de las TIC para dinamizar los procesos de enseñanza y aprendizaje" (p. 269).

También, se destaca en este estudio que la capacitación es indispensable en los procesos de enseñanza-aprendizaje en los cuales se involucra el docente, o como dicen Guayara Cuellar, Millan Rojas y Gómez Cano (2019) "la alfabetización digital" es indispensable y pertinente (p. 38); estos resultados también han sido reportados por otros estudios (Almirón y Porro, 2014; Valdés et al., 2011).

Frente a los usos de las TIC en el proceso educativo, la mayoría de los docentes está de acuerdo con el uso de estas herramientas en el aula de clase, además presentan una actitud muy favorable frente al uso de las TIC en el proceso educativo; aspecto que coincide con el estudio de Hernández Ramos y Torrijos Fincias (2019), en donde los docentes manifiestan que las TIC se "encuentran bastante integradas en sus actividades docentes" (p. 135); igualmente, con el trabajo de Laitón, Gómez, Sarmiento y Mejía (2017, p. 84). Lo anterior, igualmente es reportado por Guayara et al. (2019, p. 37), aunque en este mismo estudio también se reportó que un 33\% de los docentes no cree que las TIC sean tan importantes en los procesos de enseñanza - aprendizaje, lo que concuerda parcialmente con las percepciones de los docentes del SENA en lo que se refiere al uso de las bases de datos bibliográficas en las actividades con sus estudiantes.

Para el caso particular de los docentes indagados en este estudio, la mayoría de los mismos tienen una percepción positiva de las TIC en el campo investigativo, aunque esto no necesariamente contrastaría con una considerable producción de nuevo conocimiento por parte de estos; un estudio realizado por Gamboa et al. (2018) encontró, de hecho, que una las competencias con más bajo promedio en los docentes son las investigativas (p. 258).

Frente al uso de recursos y espacios TIC destinados por la institución, para el caso de los docentes que hicieron parte de este estudio, estos no solo los conocen, sino que también se apropian de ellos, aunque reconocen que, a veces, se tienen limitaciones, tanto de recursos como de espacios, sobre todo en los lugares descentralizados donde se imparte información. Lo anterior, contrasta con el trabajo de Lanuza Gámez, Rizo Rodríguez y Saavedra Torres (2018), quienes manifestaron en su estudio que "los docentes muestran una actitud proactiva al utilizar diferentes herramientas TIC que la universidad dispone a pesar de las limitaciones existentes en la facultad" (p. 16), pero que, al mismo tiempo, al igual que los docentes del SENA, es necesario mejorar algunos aspectos referidos a la infraestructura y a la adquisición de hardware y software. 
Finalmente, se encontró que tanto las características sociodemográficas como las del perfil profesional de los docentes no se relaciona con sus competencias TIC; ser hombre o ser mujer, de una edad u otra, con experiencia o no, con posgrado o sin él, no son factores que presenten asociación con el uso de las TIC en el ámbito educativo, o por lo menos en los docentes del centro formativo que fue estudiado; aunque en el estudio de Hernández Ramos y Torrijos Fincias (2019) se encontraron resultados similares (p. 128).

\section{| Conclusiones}

Los docentes evidencian en la capacitación un elemento fundamental para el reconocimiento de las TIC y sus elementos básicos, lo cual, además, debe ser abordado e implementado por la institución; de esta manera, la formación permanente de los docentes es también un compromiso institucional. Además, se destaca que las TIC no son solo un medio o herramienta pedagógica, sino que también se instaura como un contenido que, implícitamente, se enseña a los estudiantes; en este orden de ideas, la dinámica educativa instaura a las TIC como un elemento del currículo oculto en los programas formativos.

Las TIC son un componente importante en el proceso educativo, puesto que ayudan a ilustrar y estructurar los contenidos de la clase, asunto que, además de los docentes, es reconocido y muy bien valorado por parte de los estudiantes; en este proceso, el uso del computador, el televisor y el video beam se recalcan como herramientas comunes; se destaca el uso de la plataforma blackboard como un elemento específico y particular de la dinámica formativa del SENA (o por lo menos en el centro indagado); un elemento que tiene una percepción apenas aceptable, por parte de los docentes, es el poco uso de base de datos bibliográficas en la dinámica formativa.

A pesar de que los docentes manifiestan poseer, en términos generales, buenos elementos y formación en aspectos TIC, los cuales se usan en los procesos formativos, no siempre es posible acceder a espacios, herramientas y programas informáticos requeridos en la clase, pues no en todos los espacios, en donde se imparte formación, es posible acceder o disponer de éstos recursos; se destaca que en la sede central de la institución la posibilidad y acceso a recursos $\mathrm{TIC}$ es mejor que en otras sedes alternas.

En términos de las percepciones de los docentes frente a las TIC como un elemento facilitador de conocimiento científico, esta es muy positiva y favorable; además, que la destacan como fundamental en productos investigativos referidos a la formación de nuevos investigadores y a la producción de conferencias y comunicaciones a la comunidad educativa; no obstante, reclaman de la institución la inclusión de herramientas específicas del campo investigativo, y se reitera la necesidad de la consecución e implementación de bases de datos bibliográficas.

El uso de plataformas virtuales se destaca como uno de los recursos más importantes en los procesos formativos de la institución; no obstante, los docentes manifiestan que no siempre es posible hacerlo, dado que el SENA posee programas descentralizados, en donde las posibilidades de uso de espacios y recursos es en muchos casos limitada. Esto deja ver otro aspecto en la preparación pedagógica del docente actual; adaptarse también implica volver a lo tradicional, pues por más tecnológico y actualizado que se esté, las condiciones que a veces imperan en algunos contextos educativos no siempre favorecen la emergencia de las TIC, pero la labor educativa siempre estará presente a pesar de las circunstancias. 
Los docentes reconocen en las TIC un elemento fundamental de su perfil profesional, que connota una característica particular, y es la necesidad de estar actualizado, pues las TIC son inherentemente poco estáticas, y la evolución y avance tecnológico implican en los docentes un proceso de formación continua y constante.

En general, los docentes indagados reconocen a las TIC como un elemento fundamental (reconocimiento e implementación) en el proceso educativo e investigativo, al mismo tiempo que aluden a un conocimiento y uso de los recursos y espacios de la institución; lo anterior, se traduce en una valoración superior de sus competencias en este campo. Competencias que se asocian estadísticamente entre ellas; de lo que se puede inferir que, un buen perfil TIC en docentes de educación superior implica conocer y aplicar las TIC en la dinámica educativa, teniendo en cuenta la realidad de los sujetos y contextos en donde se interactúa. En pocas palabras, en este grupo indagado, las diferentes competencias TIC no operan por separado y se constituyen en elementos complementarios entre sí, que perfeccionan la formación permanente de los docentes; de esta manera, ser competentes en uso pedagógico de las TIC implica reconocer su utilidad inmediata y la necesidad futura de mejorarla.

Finalmente, una de las principales conclusiones que se deriva del estudio, es que los docentes reclaman que la institución sea participe de la actualización de los docentes, a partir de procesos de capacitación al interior de ella; esto denota, en gran medida, que lo relacionado con la formación permanente de los docentes, debería operar bajo un esquema de corresponsabilidad, en la medida que efectivamente la tarea de actualizarse y mantenerse al día en temáticas TIC es una preocupación del docente; pero, al mismo tiempo, también debe funcionar en términos de inversión y visión institucional, pues las herramientas, recursos y espacios tecnológicos de una institución son efectivos en la tarea educativa, si existe un agente mediador que las operacionalice y las reflexione a partir de sus competencias y destrezas.

\section{| Referencias}

Almirón, M. E. y Porro, S. (2014). Los docentes en la Sociedad de la Información: reconfiguración de roles y nuevas problemáticas. Revista Iberoamericana de Informática Educativa, (19), 17-31.

Fernández Cruz, F. J., Fernández Díaz, M. J. y Rodríguez Mantilla, J. M. (2018). El proceso de integración y uso pedagógico de las TIC en los centros educativos madrileños. Educación XX1, 21(2), 395-416. doi: 10.5944/educXX1.17907.

Gamboa Suárez, A. A., Hernández-Suárez, C. A. y Prada Núñez, R. (enero-junio, 2018). Práctica pedagógica y competencias TIC: atributos y niveles de integración en docentes de instituciones educativas de básica y media. Saber, Ciencia y Libertad, 13(1), 258-274.

González, E., Arroyave, D., Castellano, M., Urrego, A., Sepúlveda, N. y García, C. (2017). Competencias del docente siglo XXI en educación superior (informe técnico). Medellín, Colombia: Universidad de San Buenaventura.

Grisales, H. (2001). Muestreo en estudios descriptivos. Medellin, Colombia: Universidad de Antioquia. 
Guayara Cuéllar, C. T., Millan Rojas, E. E. y Gómez Cano, C. A. (2019). Diseño de un curso virtual de alfabetización digital para docentes de la Universidad de la Amazonia. Revista Científica, 1(34), 34-48. https://doi.org/10.14483/23448350.13314.

Havery López, I. C. (2014). Evaluación de un modelo de gestión de innovación en la práctica educativa apoyada en las TIC. Estudio de caso: UNIMET (Tesis de doctorado). Universidad de Sevilla. España.

Hernández Ramos, J. P. y Torrijos Fincias, P. (2019). Percepción del profesorado universitario sobre la integración de las Tecnologías de la Información y la Comunicación (TIC) en las modalidades docentes. Influencia del género y la edad. Edmetic, 8(1), 128-146. https://doi.org/10.21071/edmetic.v8i1.10537.

Hurtado Pérez, G. I. y Vélez Holguín, R. M. (2010). El papel de las TIC en la transformación del modelo escuela nueva de la Institución Educativa Octavio Calderón Mejía (Medellín, Antioquia) (Tesis de maestría). Universidad de San Buenaventura, Medellín, Colombia.

Laitón, E. V., Gómez, S. E., Sarmiento, R. E. y Mejía, C. (2017). Competencia de prácticas inclusivas: las TIC y la educación inclusiva en el desarrollo profesional docente. Sophia, 13(2), 82-95.

Lanuza Gámez F. I, Rizo Rodríguez, M. Y Saavedra Torres, L. E. (2018). Uso y aplicación de las TIC en el proceso de enseñanza-aprendizaje. Revista Científica de Farem-Estelí, 7(25), 16-30.

López, L., Correa, L. y Rojas-Bahamón, M. (2017). Representaciones sociales: formación y uso de tecnologías de información y comunicación. Profesores de educación básica secundaria. Revista Virtual Universidad Católica del Norte, (50), 256-276. Recuperado de http://revistavirtual.ucn.edu.co/index.php/RevistaUCN/article/view/823/1341

Martínez, M. (2010). Ciencia y arte en la metodología cualitativa. México: Trillas

Mesa Agudelo, W. de J. (2012). Las TIC como herramientas potenciadoras de equidad, pertinencia e inclusión educativa. Revista Trilogía, 7, 61-77.

Mesa Jiménez, F. Y. (2012). Las tecnologías de la información y la comunicación en la Universidad Colombiana: evolución y prospectiva. Revista Historia de la Educación Latinoamericana, 14(19), 71-90.

Ministerio de Educación Nacional -MEN-. (2009). Competencias Genericas en educación superior. Bogotá, Colombia: Revolución educación Colombia aprende.

Ministerio de Salud Nacional. (1993). Resolución 008430, por la cual se establecen las nomas científicas, técnicas y administrativas para la investigación en salud.

Montoya Grisales, N. E, Mosquera Bermúdes, S. P., Pérez Martínez, M. C. y Arroyave Giraldo, D. I. (2018). Competencias TIC del docente siglo XXI en educación superior. Revista Espacios, 39(53), 1-18. 
Organización de las Naciones Unidas para la Educación, la Ciencia y la Cultura - UNESCO-. (2016). Competencias y estándares TIC desde la dimensión pedagógica: una perspectiva desde los niveles de apropiación de las TIC en la práctica educativa docente. Cali, Colombia: Pontificia Universidad Javeriana.

Pérez Cervantes, M. L. y Saker, A. F. (2013). Importancia del uso de las plataformas virtuales en la formación superior para favorecer el cambio de actitud hacia las TIC; Estudio de caso: Universidad del Magdalena, Colombia. Revista Iberoamericana de Evaluación Educativa, 6(1), 153-166.

Polit, D. y Hungler, B. (2005). Investigación científica en ciencias de la salud (6á ed.). Mexico: McGraw-Hill.

Said-Hung, E., Iriarte Díaz-Granados, F., Jabba Molinares, D., Ricardo Barreto, C., Ballesteros, B., Vergara, E. y Ordóñez, M. (2015). Fortalecimiento pedagógico en las universidades en Colombia a través de las TIC. Caso región Caribe. Educación XX1, 18(2), 277-304, doi:10.5944/educXX1.14.

Sobrado, L., Ceinos, M. C. y Fernández, E. (2010). Planificación y desarrollo de un mapa de habilidades TIC en Orientación. Comunicar, 18(35), 167-174.

Strauss, A. y Corbin, J. (2002). Bases de la investigación cualitativa. Medellín, Colombia: Universidad de Antioquia.

Tedesco, J. C. (2011). Los desafíos de la educación básica en el siglo XXI. Revista Iberoamericana de Educación, 55, 31-47.

Tello Díaz, I. y Cascales Martínez, A. (2015). Las TIC y las necesidades específicas de apoyo educativo: análisis de las competencias TIC en los docentes. RIED. Revista Iberoamericana de Educación a Distancia, 18(2), 355-383. doi:https://doi.org/10.5944/ried.18.2.13536.

Valdés Cuervo, A. A., Angulo Armeta, J., Urías Martínez, M. L., García López, R. I. y Mortis Lozoya, S.V. (2011). Necesidades de capacitación de docentes de educación básica en el uso de las TIC. Píxel-Bit. Revista de Medios y Educación, (39), 211-223.

Vera Noriega, J. Á., Torres Moran, L. E. y Martínez García, E. E. (2014). Evaluación de competencias básicas en TIC en docentes de educación superior en México. Pixel-Bit, Revista de Medios y Educación, 44, 143-155. 